\title{
Pancreatitis aguda asociada a COVID-19
}

\author{
Acute pancreatitis associated with COVID-19 \\ Lorena Hernández Guadarrama, ${ }^{*, \neq}$ Javier Alvarado Durán, *,‡ \\ José Emilio Novelo Pastrana, *, Ricardo Antonio Sánchez Arteaga \\ Citar como: Hernández GL, Alvarado DJ, Novelo PJE, Sánchez ARA. Pancreatitis aguda asociada a \\ COVID-19. Acta Med GA. 2022; 20 (1): 12-16. https://dx.doi.org/10.35366/103550
}

\section{Resumen}

Introducción: En diciembre 2019 se reportó el primer caso de neumonía atípica en China, causado por SARS-CoV-2, la cual afecta principalmente el tracto respiratorio. Nuevos estudios han reportado síntomas gastrointestinales como manifestación primaria del COVID-19, pero existen pocos reportes que hayan relacionado al SARS-CoV-2 con pancreatitis aguda. Objetivo: Describir la aparición de pancreatitis y su correlación con el resto de las manifestaciones clínicas, así como considerar al páncreas como órgano blanco del virus, que pudiera empeorar el pronóstico de estos pacientes. Material y métodos: Se trata de un estudio descriptivo, trasversal, retrospectivo, no experimental de tipo serie de casos, realizado del 15 de marzo al 25 de mayo del 2020. El área de estudio corresponde a todos los pacientes que ingresaron a terapia intensiva con diagnóstico por PCR de COVID-19. Las variables estudiadas se recopilaron del expediente clínico. Resultados: Se estudiaron 30 pacientes de terapia intensiva, de los cuales ocho presentaron datos bioquímicos y tomográficos compatibles con pancreatitis aguda, todos presentaron linfopenia, elevación de amilasa y lipasa, hiperbilirrubinemia a expensas de directa; sólo un caso demostró litiasis vesicular sin ser la causa de pancreatitis, se utilizaron SOFA y APACHE para estadificar la gravedad, fallecieron todos con un promedio de 9.8 días en terapia intensiva.

Palabras clave: SARS-CoV-2, COVID-19, pancreatitis aguda, Balthazar.

* Facultad Mexicana de Medicina de la Universidad La Salle, México.

* Residente de $4^{\text {to }}$ año de Cirugía General.

$\S$ Residente $3^{\text {er }}$ año de Cirugía General.

『 Médico adscrito a Cirugía General. Hospital Angeles Clínica Londres.

Nuevo Sanatorio Durango. México.

Correspondencia:

Lorena Hernández Guadarrama

Correo electrónico: lorehgma@gmail.com

Aceptado: 14-04-2021.

www.medigraphic.com/actamedica

\section{Abstract}

Introduction: In December 2019 the first atypical pneumonia case was reported in China; caused by SARS-CoV-2. It affects mainly the respiratory tract. New studies have reported gastrointestinal symptoms as initial manifestations of COVID-19 infection and there are few reports that have linked SARS-CoV-2 to the occurrence of acute pancreatitis. Objective: To describe the appearance of pancreatitis and its correlation with the rest of the clinical manifestations, and consider the pancreas as a target organ of this virus, which could worsen the prognosis of these patients. Material and methods: This is a descriptive, cross-sectional, retrospective, non-experimental case series study conducted from March 15 to May 25, 2020. The study area corresponds to all patients who entered the intensive care area with COVID-19 PCR diagnosis. The variables studied were collected from the clinical file. Results: From thirty patients in the intensive care, eight of them presented laboratory and CT scan data compatible with acute pancreatitis. All cases have lymphopenia, the elevation of amylase and lipase, hyperbilirubinemia, and only one case showed vesicular lithiasis without demonstrating the cause of pancreatitis. It was used to stage the severity SOFA and APACHE score, all patients died in an average of days of stay in intensive care of 9.8 .

Keywords: SARS-CoV-2, COVID-19, acute pancreatitis, Balthazar.

\section{INTRODUCCIÓN}

En diciembre de 2019 se reportó el primer caso de neumonía atípica que desencadenó la pandemia por coronavirus 2019 (COVID-19) en la ciudad de Wuhan, provincia de Hubei en China, causado por el SARS-CoV-2, el cual afecta principalmente al tracto respiratorio, la incidencia del síndrome de dificultad respiratoria aguda (SDRA) fue de $19.5 \%,{ }^{1}$ los síntomas más comunes fueron fiebre (80.4\%), fatiga $(46 \%)$, tos seca $(63.1 \%)$, expectoración $(41.8 \%)$ y disnea (31\%); así mismo, nuevos estudios han reportado síntomas gastrointestinales como manifestación primaria 
del COVID-19. Por otra parte, existen actualmente pocos reportes que hayan relacionado al SARS-CoV-2 con la aparición de pancreatitis aguda en el contexto de esta enfermedad. ${ }^{2}$

El actual brote de la enfermedad COVID-19 causada por el coronavirus del síndrome respiratorio agudo severo (SARS-CoV-2) ha desencadenado una crisis mundial de salud pública. Actualmente, en México, para el 18 de julio 2020 , se cuenta con 338,913 casos confirmados, con total de defunciones de 40,762 (10.9\%). ${ }^{3}$

La pancreatitis aguda se refiere a una inflamación aguda del tejido pancreático, ${ }^{4}$ es uno de los principales motivos de admisión hospitalaria a nivel mundial. La etiología es diversa, siendo las causas más comunes el origen biliar, el abuso de alcohol, la hipertrigliceridemia y el uso de algunos fármacos. Estudios recientes han mostrado una etiología viral por Paramyxoviridae, Coxsackie, hepatitis B, citomegalovirus, varicela zóster, herpes simple y virus de la inmunodeficiencia humana. ${ }^{5}$ La pancreatitis aguda representa un espectro de enfermedades que van desde un curso leve y autolimitado que requiere sólo una breve hospitalización, hasta una enfermedad fulminante y rápidamente progresiva que da lugar al síndrome de disfunción orgánica múltiple, con o sin la sepsis que lo acompaña. Estudios previos han demostrado que el SARS-CoV-2 infecta a las células mediante la unión a la enzima convertidora de angiotensina 2 (ECA2). ${ }^{6}$

Estas proteínas de ECA2 son expresadas abundantemente en la superficie apical del epitelio ciliar de los pulmones (células alveolares tipo 2), enterocitos del intestino delgado, además de células endoteliales arteriales y venosas, en el tejido cardiaco, renal, glándulas adrenales y páncreas, confiriendo así un sitio blanco para infección COVID-19.7 Al mismo tiempo, el daño pancreático posterior a la infección por SARS-CoV-2 no ha sido apropiadamente referido; hay una falta de atención en la lesión pancreática que puede acelerar la progresión de los pacientes. En nuestra observación clínica, también encontramos que algunos mostraron signos de lesión pancreática, como altos niveles de amilasa y lipasa en sangre. También se cree que la lesión pancreática podría ser causada indirectamente por respuestas sistémicas a la insuficiencia respiratoria o por la respuesta inmunológica perjudicial inducida por la infección por el SARS-CoV-2, que también provocó el daño en múltiples órganos. ${ }^{8}$

A continuación, presentamos ocho casos de infección por COVID-19 con diferentes grados de severidad, atendidos en nuestra institución, en los cuales se encuentra la presencia de pancreatitis aguda como común denominador.

El objetivo de este trabajo es describir la aparición de esta entidad y su correlación con el resto de las manifestaciones clínicas de esta enfermedad, así como considerar al páncreas como otro potencial órgano blanco de este virus, lo que podría empeorar el pronóstico de estos pacientes, como factor independiente de las manifestaciones respiratorias ya descritas.

\section{REPORTE DE CASOS}

Un grupo de ocho pacientes, quienes se encontraban bajo el cuidado del servicio de la Unidad de Cuidados Intensivos $(\mathrm{UCl})$, con PCR para SARS-CoV-2 positiva en todos los casos, fueron reportados con datos clínicos y por imagen de pancreatitis aguda, teniendo una duración de estancia en dicho servicio de entre tres y 17 días, con un índice de masa corporal (IMC) promedio de $32.5 \mathrm{~kg} / \mathrm{m}^{2}$, la mitad del grupo contaba con el antecedente de hipertensión arterial sistémica, 25\% con antecedente de diabetes mellitus tipo 2, todos con diagnóstico de obesidad, 50\% grado I, 25\% grado

\begin{tabular}{cccccc}
\multicolumn{5}{c}{ Tabla 1: Datos antropométricos y antecedentes personales patológicos } \\
de pacientes con neumonía atípica por COVID-19.
\end{tabular}


Tabla 2: Hallazgos de laboratorios al ingreso.

\begin{tabular}{|c|c|c|c|c|c|c|c|c|c|c|c|c|c|c|c|}
\hline P & $\begin{array}{c}\text { Leucocitos } \\
\left(10^{3} / \mu \mathrm{L}\right)\end{array}$ & $\begin{array}{c}\text { Neutrófilos } \\
(\%)\end{array}$ & $\begin{array}{c}\text { Linfocitos } \\
(\%)\end{array}$ & $\begin{array}{l}\text { Plaquetas } \\
\left(10^{3} / \mu \mathrm{L}\right)\end{array}$ & $\begin{array}{c}\text { Creatinina } \\
(\mathrm{mg} / \mathrm{dL})\end{array}$ & $\begin{array}{l}\text { Glucosa } \\
\text { (mg/dL) }\end{array}$ & $\begin{array}{l}\text { LDH } \\
(\mathrm{U} / \mathrm{L})\end{array}$ & $\begin{array}{c}\text { BT } \\
(\mathrm{mg} / \mathrm{dL})\end{array}$ & $\begin{array}{c}\mathrm{BD} \\
(\mathrm{mg} / \mathrm{dL})\end{array}$ & $\begin{array}{c}\mathrm{BI} \\
(\mathrm{mg} / \mathrm{dL})\end{array}$ & $\begin{array}{l}\text { TGO } \\
(\mathrm{U} / \mathrm{L})\end{array}$ & $\begin{array}{l}\text { TGP } \\
(\mathrm{U} / \mathrm{L})\end{array}$ & $\begin{array}{c}\text { FA } \\
(\mathrm{U} / \mathrm{L})\end{array}$ & $\begin{array}{c}\text { Amilasa } \\
(\mathrm{U} / \mathrm{L})\end{array}$ & $\begin{array}{c}\text { Lipasa } \\
(\mathrm{U} / \mathrm{L})\end{array}$ \\
\hline 1 & 5,640 & 76 & 18 & 160,000 & 0.6 & 95 & 453 & 3.4 & 2.6 & 0.8 & 50 & 234 & 93 & 375 & 1,903 \\
\hline 2 & 5,750 & 81 & 12 & 116,000 & 0.7 & 145 & 429 & 5.5 & 4.6 & 0.9 & 568 & 67 & 371 & 434 & 2,813 \\
\hline 3 & 11,900 & 92 & 6 & 171,000 & 1.4 & 110 & 570 & 4.6 & 3.2 & 1.4 & 72 & 38 & 114 & 230 & 1,230 \\
\hline 4 & 8,510 & 85 & 9 & 209,000 & 1.6 & 175 & 889 & 2.3 & 1.6 & 0.7 & 53 & 37 & 48 & 266 & 1,163 \\
\hline 5 & 10,740 & 66 & 17 & 181,000 & 1.7 & 87 & 1,585 & 5.5 & 2.7 & 2.8 & 1,389 & 2,087 & 257 & 700 & 1,254 \\
\hline 6 & 12,098 & 95 & 15 & 162,000 & 1.3 & 100 & 678 & 4.9 & 2.0 & 2.9 & 1,233 & 2,322 & 432 & 948 & 2,340 \\
\hline 7 & 9,540 & 86 & 16 & 250,000 & 1.5 & 143 & 949 & 5.3 & 3.0 & 2.3 & 932 & 1,421 & 98 & 215 & 3,453 \\
\hline 8 & 11,403 & 87 & 13 & 90,000 & 1.1 & 254 & 566 & 3.2 & 1.9 & 1.3 & 674 & 987 & 121 & 245 & 1,176 \\
\hline
\end{tabular}

$\mathrm{P}=$ paciente; $\mathrm{LDH}=$ lactato deshidrogenasa; $\mathrm{BT}=$ bilirrubinas totales; $\mathrm{BD}=$ bilirubina directa; $\mathrm{BI}$ = bilirrubina indirecta, $\mathrm{TGO}=$ transaminasa glutámico oxalacética; TGP = transaminasa glutámico pirúvica; FA = fosfatasa alcalina.

Tabla 3: Características de pacientes con neumonía por COVID-19 con pancreatitis aguda.

\begin{tabular}{|c|c|c|c|c|c|c|c|c|c|c|c|}
\hline$P$ & $\begin{array}{c}\text { Síntomas } \\
\text { GI }\end{array}$ & Fiebre & $\begin{array}{l}\text { RT PCR } \\
\text { (SARS } \\
\text { CoV-2) }\end{array}$ & TAC de tórax & PACHE* & SOFA $^{* *}$ & $\begin{array}{l}\text { Ingreso } \\
\text { con diag- } \\
\text { nóstico de } \\
\text { pancrea- } \\
\text { titis }\end{array}$ & $\begin{array}{l}\text { TAC de } \\
\text { abdomen }\end{array}$ & PCR & $\begin{array}{l}\text { Procalcito- } \\
\text { nina }\end{array}$ & Falleció \\
\hline 1 & Diarrea & Sí & Positiva & CO-RADS 5 & 21 & 10 & No & Balthazar C & 31.9 & 2.93 & Sí \\
\hline 2 & No & Sí & Positiva & CO-RADS 5 & 23 & 13 & No & No & 805.0 & 5.70 & Sí \\
\hline 3 & Diarrea & Sí & Positiva & CO-RADS 5 & 22 & 11 & No & Balthazar C & 410.0 & 6.73 & Sí \\
\hline 4 & No & Sí & Positiva & CO-RADS 5 & 25 & 6 & Sí & Balthazar A & 231.0 & 3.30 & Sí \\
\hline 5 & STDA & Sí & Positiva & CO-RADS 5 & 24 & 9 & No & No & 45.0 & 1.03 & Sí \\
\hline 6 & Diarrea & Sí & Positiva & CO-RADS 5 & 25 & 10 & Sí & Balthazar C & 234.0 & 2.30 & Sí \\
\hline 7 & Diarrea & Sí & Positiva & CO-RADS 5 & 23 & 14 & No & Balthazar B & 123.0 & 2.94 & Sí \\
\hline 8 & STDA & Sí & Positiva & CO-RADS 5 & 29 & 15 & Sí & Balthazar C & 432.0 & 2.03 & Sí \\
\hline
\end{tabular}

$\mathrm{GI}$ = gastrointestinales; STDA = sangrado de tubo digestivo alto; RT PCR = reacción en cadena de la polimerasa con transcriptasa inversa; TAC = tomografía axial computarizada; CO-RADS = Coronavirus Disease 2019 Reporting and Data System; APACHE = Acute Physiology And Chronic Health Evaluation; SOFA = Sequential Organ Failure Assessment; $P C R=$ proteína C reactiva.

*APACHE (puntos): 20-24 mortalidad de 40\%, 25-29 mortalidad de 55\%.

**SOFA (puntos): 6-7 mortalidad de 21.5\%, 8-9 mortalidad de 33.3\%, 10-11 mortalidad de 50\%, 12-14 mortalidad de 80\%, > 14 mortalidad de $95.2 \%$.

II; sólo de 37.5\% se integró el diagnóstico de pancreatitis aguda a su ingreso (Tabla 1).

Todos requirieron manejo avanzado de la vía aérea con ventilación mecánica debido a falla respiratoria aguda; manejo con aminas vasoactivas, seis pacientes con norepinefrina/dobutamina y dos con norepinefrina únicamente; reanimación hídrica, así como tratamiento en todos los casos con ritonavir e ivermectina (tres dosis) y dexametasona como antiinflamatorio. Todos los pacientes presentaron fiebre durante su estancia, un paciente presentó sangrado de tubo digestivo bajo y tres pacientes diarrea como manifestaciones gastrointestinales.

Se tomaron paraclínicos al ingreso al servicio en los que se encontró leucocitosis en la mitad de los casos, linfopenia en $12.5 \%$ y trombocitopenia en $25 \%$. El $62.5 \%$ tuvo retención de azoados y lesión renal aguda, hiperglucemia, teniendo como promedio $180 \mathrm{mg} / \mathrm{dL}$, elevación de deshidrogenasa láctica en todos los casos. En cuanto a las pruebas de función hepática, las bilirrubinas totales se encontraban elevadas en todos los casos, mostrando patrón obstructivo en dos casos, mixto en cuatro y datos de daño hepático 
en cinco pacientes, la amilasa y lipasa se encontraban elevadas por encima de tres veces su valor normal. Como marcador de inflamación se utilizó la proteína $\mathrm{C}$ reactiva, la cual estaba elevada en todos los casos, a excepción de dos; procalcitonina elevada en los ocho pacientes (Tabla 2).

Durante su estancia se estadificó por tomografía a todos los casos de neumonía atípica, otorgando un puntaje CORADS 5 para los ocho. Se realizó tomografía abdominal a todos los casos otorgando clasificación Balthazar C en cuatro casos, un caso A y un caso B (Tabla 3).

Por otra parte, las escalas pronósticas utilizadas al ingreso a la $\mathrm{UCl}$ de estos pacientes fueron: APACHE II (acrónimo en inglés de Acute Physiology And Chronic Health Evaluation), obteniendo un puntaje promedio de 23.9, lo que se traduce como $40 \%$ de mortalidad no operatoria y SOFA (Sequential Organ Failure Assessment) puntaje de 10.62, lo que significa $50 \%$ de mortalidad.

A pesar del manejo con soporte respiratorio mecánico invasivo, apoyo vasopresor y tratamiento médico indicado, el desenlace en todos los casos fue de mortalidad de $100 \%$. Durante la recolección de datos se observó que los pacientes presentan datos de toxicidad a nivel hepático secundario a tratamiento con múltiples fármacos hepatotóxicos, principalmente: ivermectina, causante de alteraciones hepáticas con elevación de transaminasa glutámico oxalacética (TGO) 2\%, transaminasa glutámico pirúvica (TGP) 1\%, recibiendo dos dosis, que es la máxima utilizada en otras infecciones graves (estrongiloidiasis), azitromicina que puede ocasionar elevación de TGP $<6 \%$, fluconazol que puede causar hepatitis fulminante, alteración de transaminasas en frecuencia no definida, lopinavir/ ritonavir elevación de enzimas hepáticas (TGP grado 3/4) hasta $11 \%$, siendo estos dos últimos los más probablemente relacionados con estas alteraciones hepáticas. ${ }^{9}$

\section{DISCUSIÓN}

Se estudiaron 30 pacientes que ingresaron al área de cuidados intensivos con diagnóstico por PCR de COVID-19, en el periodo que abarca del 15 de marzo al 25 de mayo del 2020, de los cuales ocho presentaron datos bioquímicos y tomográficos compatibles con pancreatitis aguda. Se observó que la incidencia de COVID-19 es mayor en varones que en mujeres, para lo que se ha propuesto como posible explicación la influencia del cromosoma $\mathrm{X}$ y las hormonas sexuales en la inmunidad innata y adaptativa. La media de edad fue de 50 años, el índice de masa corporal promedio fue de 32.6, sólo en un caso se demostró que el paciente tuvo litiasis vesicular sin demostrar que fuera la causa de pancreatitis.

En nuestra serie de casos de 30 pacientes ingresados en terapia intensiva con diagnóstico de ingreso por neumonía atípica por COVID-19, se encontró que 26.6\% presentó cuadro de pancreatitis aguda. En comparación con una serie de casos de 52 pacientes de COVID-19, donde se informó que la lesión pancreática estaba presente en 17\% de los pacientes. ${ }^{10}$ No se ha informado de ningún caso de pancreatitis aguda causada por el SARS-CoV-2. Aunque, hay informes de otros coronavirus que causan pancreatitis en animales.

Dada la disociación temporal entre la pancreatitis y el resto del cuadro clínico, sugerimos que la afectación pancreática probablemente surge de una respuesta inflamatoria mediada por el sistema inmunológico, en lugar de una lesión viral directa, aunque la ECA2 está altamente expresada en el tejido pancreático.

Informamos de ocho casos de pancreatitis aguda en pacientes con COVID-19, destacando la importancia de considerar el SARS-CoV-2 como un nuevo agente etiológico de la pancreatitis viral aguda. Se necesitan más estudios para establecer la verdadera prevalencia y la importancia clínica de la lesión pancreática en los pacientes con COVID-19.

\section{CONCLUSIONES}

Aunque se desconoce la patogénesis clara, la pancreatitis aguda en COVID-19 podría ocurrir debido al efecto citopático directo de la replicación local del SARS-CoV-2 o indirectamente por la respuesta inmunológica perjudicial inducida por el virus. No obstante, nuestra comprensión de las manifestaciones clínicas de COVID-19 sigue progresando, y demostramos que el SARS-CoV-2 puede precipitar pancreatitis aguda en el entorno de COVID-19. Por otra parte, la COVID-19 también puede provocar falla multiorgánica, por lo tanto, no es posible evaluar si la pancreatitis aguda contribuye al curso grave de la enfermedad.

Por lo anterior, es importante evaluar las enzimas pancreáticas en pacientes hospitalizados con síntomas gastrointestinales, ya que podrían revelar una afectación pancreática no reconocida en esta población, y excluir otras causas de pancreatitis aguda en los pacientes (alcohol, obstrucción biliar/cálculos biliares, drogas, traumatismos, hipertrigliceridemia e hipercalcemia). Estos casos destacan la pancreatitis aguda como una complicación asociada con COVID-19 y subrayan la importancia de medir enzimas pancreáticas en pacientes con COVID-19, especialmente si los pacientes experimentan dolor abdominal.

\section{REFERENCIAS}

1. Xu B, Kraemer MUG; Open COVID-19 Data Curation Group. Open access epidemiological data from the COVID-19 outbreak. Lancet Infect Dis. 2020; 20 (5): 534. 
2. Chen N, Zhou M, Dong X, Qu J, Gong F, Han Y et al. Epidemiological and clinical characteristics of 99 cases of 2019 novel coronavirus pneumonia in Wuhan, China: a descriptive study. Lancet. 2020; 395 (10223): 507-513.

3. Dirección General de Epidemiología. Secretaría de Salud [Internet]. Datos abierto dirección general de epidemiología. Información importante Coronavirus COVID-19. 2020 [citado 8 julio 2020]. Disponible en: https://www.gob.mx/salud/documentos/datos-abiertos-152127

4. Lankisch PG, Apte M, Banks PA. Acute pancreatitis. Lancet. 2015; 386 (9988): 85-96.

5. Rawla P, Bandaru SS, Vellipuram AR. Review of infectious etiology of acute pancreatitis. Gastroenterology Res. 2017; 10 (3): 153-158.

6. Dalan R, Bornstein SR, El-Armouche A, Rodionov RN, Markov A, Wielockx B et al. The ACE-2 in COVID-19: foe or friend? Horm Metab Res. 2020; 52 (5): 257-263.
7. Liu F, Long X, Zhang B, Zhang W, Chen X, Zhang Z. ACE2 Expression in pancreas may cause pancreatic damage after SARS-CoV-2 infection. Clin Gastroenterol Hepatol. 2020; 18 (9): 2128-2130.

8. Baron M, Veres A, Wolock SL, Faust AL, Gaujoux R, Vetere A et al. A Single-cell transcriptomic map of the human and mouse pancreas reveals inter- and intra-cell population structure. Cell Syst. 2016; 3 (4): 346-360.

9. Oyelade T, Alqahtani J, Canciani G. Prognosis of COVID-19 in patients with liver and kidney diseases: an early systematic review and metaanalysis. Trop Med Infect Dis. 2020; 5 (2): 80.

10. Wang F, Wang H, Fan J, Zhang Y, Wang H, Zhao Q. Pancreatic injury patterns in patients with coronavirus disease 19 pneumonia. Gastroenterology. 2020; 159 (1): 367-370. 